\title{
A GESTÃO DO CAPITAL INTELECTUAL ATRAVÉS DAS UNIVERSIDADES
} CORPORATIVAS

\author{
Kamilla Da Silva Ribeiro' \\ Prof. M. Sc. Rickardo Léo Ramos Gomes ${ }^{2}$ \\ rickardolrg@yahoo.com.br \\ https://orcid.org/0000-0001-6101-9571
}

\begin{abstract}
Para citar este artículo puede utilizar el siguiente formato:
Kamilla Da Silva Ribeiro y Rickardo Léo Ramos Gomes: "Gestión del capital intelectual a través de universidades corporativas", Revista Contribuciones a las Ciencias Sociales, (Vol 1, № 8 octubrediciembre 2021, pp. 433-443). En línea:
\end{abstract}

https://doi.org/10.51896/CCS/ORES5314

\section{RESUMO}

Investigou-se neste artigo sobre a gestão do capital intelectual por meio das universidades corporativas. $\mathrm{Na}$ era da informação, a gestão do conhecimento é fator preponderante para o alcance da vantagem competitiva na economia global através do desenvolvimento de ferramentas como inteligência artificial, gerenciamento da informação, entre outros. O objetivo geral deste artigo é: Investigar sobre a gestão do capital intelectual através das universidades corporativas. Os objetivos específicos determinados foram os seguintes: explicar sobre a gestão do capital intelectual através das universidades corporativas e analisar várias citações estudadas de várias obras de autores diferentes. A metodologia empregada caracteriza-se por uma abordagem qualitativa constituída por uma pesquisa bibliográfica quando foram pesquisados referenciais teóricos publicados tais como livros, artigos científicos e documentos para um melhor esclarecimento do tema abordado. Verificase que não existe ameaça das universidades corporativas às universidades tradicionais, tendo em vista a parceria existente entre as duas instituições para o desenvolvimento de competências

1 Graduada em Administração de Empresas pela Universidade de Fortaleza; Pós-graduada em Administração Hospitalar pela Faculdade Gama Filho; Pós-graduanda em MBA em Gestão de Pessoas pelo Centro Universitário Farias Brito (FBUNI); Coordenadora Técnica na Fundação Bradesco.

2 Prof. da Disc. de Met. do Trabalho Científico (Orientador) - Inst. Euvaldo Lodi; C. U. UniAteneu; C. U. Farias Brito; M. Sc. em Fitotecnia pela Universidade Federal do Ceará (UFC); Spec. em Met. do Ens. de Ciências pela UECe; Grad. em Agronomia pela UFC; Licenciado na Área de Ciências da Natureza, Matemática e suas Tecnologias pela UVA; Aperf. em Líderes de Aprendizagem pela Universidade de Harvard; Aperf. em Gestão de Riscos em Projetos pelo BID; Aperf. em Met. do Trabalho Científico pela FIOCRUZ. Curso Aperf. Rastreamento do Contato da COVID-19 pela Johns Hopkins University (JHBSPH); Consultor Internacional do BIRD para Laboratórios Científicos. Fundador da RLRG Consultoria Científica. 
necessárias para o mercado de trabalho, enquanto existir atualização contínua, existirá desenvolvimento e atuação para ambas.

Palavras-chave: Universidades Corporativas. Gestão do Conhecimento. Capital Intelectual.

\section{GESTIÓN DEL CAPITAL INTELECTUAL A TRAVÉS DE UNIVERSIDADES CORPORATIVAS}

\section{RESUMEN}

Este artículo investiga la gestión del capital intelectual a través de universidades corporativas. En la era de la información, la gestión del conocimiento es un factor clave para lograr una ventaja competitiva en la economía global mediante el desarrollo de herramientas como la inteligencia artificial, la gestión de la información, entre otras. El objetivo general de este artículo es: Investigar la gestión del capital intelectual a través de universidades corporativas. Los objetivos específicos determinados fueron los siguientes: explicar sobre la gestión del capital intelectual a través de universidades corporativas y analizar varias citas estudiadas de varios trabajos de diferentes autores. La metodología utilizada se caracteriza por un abordaje cualitativo consistente en una investigación bibliográfica cuando se buscaron referencias teóricas publicadas como libros, artículos científicos y documentos para una mejor aclaración del tema abordado. Parece que no existe una amenaza de las universidades corporativas a las universidades tradicionales, en vista de la asociación existente entre las dos instituciones para el desarrollo de las habilidades necesarias para el mercado laboral, mientras haya una actualización continua, habrá desarrollo y acción para ambos.

Palabras clave: Universidades corporativas. Conocimiento administrativo. Capital intelectual.

\section{MANAGEMENT OF INTELLECTUAL CAPITAL THROUGH CORPORATE UNIVERSITIES}

\section{ABSTRACT}

This article investigates the management of intellectual capital through corporate universities. In the information age, knowledge management is a major factor in achieving competitive advantage in the global economy through the development of tools such as artificial intelligence, information management, among others. The general objective of this article is: To investigate the management of intellectual capital through corporate universities. The specific objectives determined were: to explain about the management of intellectual capital through corporate universities and to analyze several studied citations of several works by different authors. The methodology used is characterized by a qualitative approach consisting of a bibliographic research when published theoretical references such as books, scientific articles and documents were searched for a better clarification of the topic addressed. It appears that there is no threat from corporate universities to traditional universities, in view of the existing partnership between the two institutions for the development of skills necessary for the labor market, as long as there is continuous updating, there will be development and action for both.

Keywords: Corporate University. Knowledge management. Intellectual capital. 


\section{INTRODUÇÃO}

O ativo principal das organizações contemporâneas é o capital humano. Eles precisam gerenciar a si mesmo e ter autonomia. Dessa forma, é natural que sejam estimulados a enfrentar os desafios, para a criação do potencial corporativo. Na era da informação, a gestão do conhecimento é fator preponderante para o alcance da vantagem competitiva na economia global através do desenvolvimento de ferramentas como inteligência artificial, gerenciamento da informação, entre outros. Na visão da nova gestão de conhecimento entendem que o conhecimento é um processo, baseado em competências, habilidades e atitudes (Araújo, 2018).

Para Eboli (2012), a universidade corporativa atua no desenvolvimento da gestão organizacional por meio de métodos de aprendizagem, enquanto os centros tradicionais de T\&D (Treinamento e Desenvolvimento) desenvolvem pessoas para situações específicas. O presente artigo investigou sobre temas relativos à gestão do capital intelectual por meio das universidades corporativas. Procurou investigar se esse modelo educacional vem contribuindo para 0 aperfeiçoamento dos funcionários e da organização de forma ampla e assertiva. A educação corporativa atua com objetivo de desenvolver os colaboradores e parceiros de negócios nas competências relacionadas às estratégias da organização, são veículos de disseminação da cultura por proporcionar a vivência real das empresas.

O objetivo geral deste artigo é: Investigar sobre a gestão do capital intelectual através das universidades corporativas. Os objetivos específicos determinados foram os seguintes: explicar sobre a gestão do capital intelectual através das universidades corporativas e analisar várias citações estudadas de várias obras de autores diferentes.

A metodologia empregada caracteriza-se por uma abordagem qualitativa constituída por uma pesquisa bibliográfica quando foram pesquisados referenciais teóricos publicados tais como livros, artigos científicos e documentos para um melhor esclarecimento do tema abordado.

Este artigo foi estruturado da seguinte forma: No primeiro tópico apresentou-se a introdução destacando os objetivos desta pesquisa. No segundo tópico foram destacados os procedimentos metodológicos empregados nesta investigação. No terceiro tópico promoveu-se uma discussão teórica e no quarto e último tópico foram elaboradas as considerações finais.

\section{METODOLOGIA}

O presente trabalho seguiu uma abordagem qualitativa com caráter exploratório e descritivo e a metodologia empregada foi a da pesquisa bibliográfica. (HUHNE, 1999) Para tanto foram analisadas várias contribuições culturais e científicas relacionadas com o assunto em questão. Brizolla et al (2020, p. 111) explicam que

A pesquisa qualitativa é uma atividade situada que localiza o observador no mundo. Trata-se 
de um conjunto de práticas interpretativas que tornam o mundo visível. Essas práticas transformam 0 mundo. A pesquisa qualitativa transforma 0 mundo em uma série de representações, incluindo notas de campo, entrevistas, conversas, fotografias, gravações e memorandos etc. A este nível, a pesquisa qualitativa envolve uma abordagem interpretativa, naturalista ao assunto em questão. Isso significa que os pesquisadores qualitativos estudam as coisas em seus ambientes naturais, tentando dar sentido ou interpretar fenômenos em termos dos significados que as pessoas trazem para eles.

Desta maneira procurou-se retirar e analisar múltiplas citações estudadas de várias obras de autores diferentes, dos quais destacam-se: Leal et al. (2018); Silva (2018), Brito e Castro (2019); Pessoa, Felix e Gomes (2019); Vidal e Miguel (2020); Aguilera (2020); Silva, Freitas e D'ambrosio (2021). Assim, os posicionamentos tomados no decorrer do artigo, foram fundamentados pelas opiniões dos autores consultados, aqui compartilhadas, e que serviram de referencial para a formação do corpus do presente texto. (Huhne, 1999)

\section{DISCUSSÃO}

Esta fundamentação foi ordenada em três subtópicos. No primeiro falou-se sobre gestão do conhecimento e processo de aprendizagem nas UCs, tendo como objetivo apesentar um suporte teórico relacionado ao tema Universidade Corporativa (UC), trazendo a compreensão do tema. No segundo subtópico, discorreu-se sobre as mudanças no paradigma de T\&D para a UC e no terceiro subtópico foi abordada a atuação das universidades corporativas em relação as universidades tradicionais dentro do contexto de oportunidade ou ameaças, o quanto as novas tecnologias e mecanismos de aprendizagem juntos, contribuem para o desenvolvimento.

\subsection{Gestão do Conhecimento e Aprendizagem nas Universidades Corporativas}

Dentro do cenário atual de competitividade, a gestão do conhecimento atua como um recurso estratégico inserido no cotidiano das pessoas. Abrange o capital intelectual, capital humano, a nossa capacidade de pesquisar e inovar nas organizações. Na opinião de Silva (2018, p. 25)

A preocupação com a gestão do conhecimento dos colaboradores das organizações está, cada vez mais, presente no cotidiano e se materializa com a implantação de universidades corporativas. Os grandes desafios das empresas hoje exigem foco na performance e na criação de ambientes de aprendizagem contínuo. O que antes não era tão importante passou a ter um peso imensurável, a educação. Atualmente o mundo vive na gestão do conhecimento, quanto mais bem preparado e mais informações, mais rico é o seu intelecto empresarial.

O ativo principal das organizações contemporâneas é o capital humano. Eles precisam gerenciar a si mesmo e ter autonomia. Dessa forma, é natural que sejam estimulados a enfrentar os 
desafios, para a criação do potencial corporativo. Falando sobre gestão de conhecimento Pessoa, Félix e Gomes (2019, p. 08) afirmam:

[...] a gestão de conhecimentos que é produzido pelas empresas, está relacionada aos processos que devem estar mapeados, para garantir o conhecimento adquirido com a execução das atividades, buscando diminuir o tempo com treinamentos e capacitação com novos colaboradores.

Essa gestão do conhecimento se faz necessária dentro das organizações, principalmente, por conta dos departamentos e processos que são realizados diariamente, favorecendo a empresa como vantagem competitiva na redução de custos com planejamentos efetivos, novos modelos de negócios e o desenvolvimento do seu capital intelectual. Silva, Nascimento, Silva e Gomes (2018, p. 05) consideram importante esclarecer sobre a educação corporativa antes de aprofundar sobre a temática das universidades corporativas propriamente dita:

\begin{abstract}
A educação corporativa é um plano de desenvolvimento de carreira dos colaboradores a curto e longo prazo, que deve ser implantado conforme as diretrizes estratégicas da organização, com o objetivo de aumentar seu capital intelectual e promover o crescimento de seus colaboradores. Ao implantarmos um plano de educação corporativa, é necessário primeiramente realizar um planejamento junto às lideranças para que possam ser traçadas as necessidades de qualificação das equipes, deve-se levar em consideração a cultura da empresa, sua história, realizar a análise do perfil organizacional e do colaborador.
\end{abstract}

Falsarella e Jannuzzi (2017) ressaltam que é necessário planejar o futuro sempre considerando bases confiáveis e concretas, longe de intuições sem fundamentação, de modo que a organização consiga sobreviver à competitividade infligida pelo mercado, que faz, constantemente, a organização precisar de novas e aceleradas respostas. O foco da aprendizagem deve ocorrer em todos os níveis da organização. É assim que o conhecimento se transforma em competências através da mobilização, aprendizagem, participação e comprometimento. O conhecimento é inerente ao indivíduo. É responsabilidade da organização apoiar os colaboradores criativos para a criação do conhecimento (Araújo, 2018)

Dentro desse contexto, identifica-se que a aprendizagem na universidade corporativa é alinhada e planejada de acordo com os objetivos estratégicos da organização, sendo necessário ser contínuo e sistemático para ser eficiente. Aprender a aprender faz com que o indivíduo possa lidar com o novo, mudanças e sempre buscar o autodesenvolvimento, melhorar constantemente buscando novas técnicas e competências nas organizações. O indivíduo mesmo com suas qualificações, tem que estar sempre buscando o aprendizado contínuo, para o desenvolvimento de suas atribuições e responsabilidades (Araújo, 2018; Fleury; Fleury, 2002 apud Leal et al., 2018).

\title{
3.1.1 Universidades Corporativas
}


Segundo Gould (2005), a universidade corporativa é um modelo educacional contemporâneo onde a responsabilidade pela educação é transferida da academia para o setor empresarial e é onde, também, se discute e desenvolve soluções para desenvolvimento de competências com foco nos objetivos organizacionais.

Esse modelo difere do modelo educacional tradicional, pois é projetado para alunos adultos e é de duração longa e não de duração limitada, pois tem como finalidade o desenvolvimento ao longo de uma carreira dentro da organização. O currículo é dirigido pelo mercado e é projetado para manter uma força de trabalho especializada. Portanto, a educação, nesse caso, é para desenvolvimento profissional e não pessoal (Gould, 2005 citado por Araújo, 2018)

Meister (1999) citado por Esteves; Meiriño (2016, p.14) afirma que "a Universidade Corporativa (UC) alinha os objetivos empresariais de todos os envolvidos nas organizações, e com base na estratégia organizacional, oferece soluções de aprendizagem voltada para cada conjunto funcional”. Já Barley (2002), afirma que a UC serve para apoiar a missão das empresas, manter a competitividade, selecionar talentos, desenvolver ações estratégicas, satisfazer os clientes, orientar mudanças organizacionais e desenvolver competências.

Eboli (2012) afirma que as UC's, também, têm o dever de desenvolver a vontade de aprender dos colaboradores e ensiná-los a gerenciar seus conhecimentos. $O$ ambiente interno das organizações é impactado pelo ambiente externo, diante desse cenário, para acompanhar as exigências da competitividade, é necessário desenvolver os colaboradores aprimorando os seus conhecimentos, habilidades e competências.

A Educação corporativa atua com objetivo de desenvolver os colaboradores e parceiros de negócios nas competências relacionadas às estratégias da organização, são veículos de disseminação da cultura por proporcionar a vivência real das empresas. O grande objetivo das universidades corporativas é oferecer cursos específicos aos funcionários, sendo assim, hoje, é possível criar dentro do universo corporativo cursos e treinamentos específicos, esses profissionais são design instrucionais que contribuem, fortemente, personalizando conteúdos de acordo com as necessidades estratégicas do negócio.

\subsection{Mudanças no Paradigma de Treinamento e Desenvolvimento (T\&D) para a Universidade Corporativa}

Treinamento e desenvolvimento de pessoas são processos de capacitação de muita importância dentro da gestão de pessoas, tratando iniciativas pontuais, de curto prazo e práticas de longo prazo, visando sempre o crescimento profissional. Para Eboli (2012), a universidade corporativa atua no desenvolvimento da gestão organizacional por meio de métodos de aprendizagem, enquanto os centros tradicionais de T\&D (Treinamento e Desenvolvimento) desenvolvem pessoas para situações específicas. 
Segundo Pereira (2010), aponta que o sistema T\&D está focado no melhoramento 23 das habilidades do colaborador para realização de suas tarefas, ou seja, concentra-se mais no aprendizado individual, gerando, assim, aumento das habilidades. Meister (1999) acrescenta que a diferença da UC está na dimensão, no público-alvo, pois incluem funcionários, clientes, fornecedores. Allen (2002), afirma que, para se ter uma universidade corporativa, as atividades educacionais devem ter um vínculo claro com as estratégias organizacionais. A seguir no quadro 1 é possível evidenciar a diferença que existe entre o método de aprendizagem de T\&D (Desenvolvimento e treinamento), para Universidades Corporativas.

\section{Quadro 1:}

Mudança de Paradigma do Centro de Treinamento para a Unidade Corporativa

\begin{tabular}{|c|c|c|}
\hline \multicolumn{3}{|c|}{ MUDANÇA DE PARADIGMA } \\
\hline $\begin{array}{c}\text { CENTRO DE } \\
\text { TREINAMENTO }\end{array}$ & $\begin{array}{c}\text { UNIDADE DE EDUCACAO } \\
\text { CORPORATIVA }\end{array}$ \\
\hline Reativo & AÇÃO & Proativo \\
\hline Desenvolver Habilidades & OBJETIVO & Desenvolver Competências \\
\hline Aprendizagem Individual & FOCO & Aprendizagem organizacional \\
\hline Tático & ESCOPO & Estratégico \\
\hline Necessidades Individuais & ÉNFASE & Necessidades Organizacionais \\
\hline Interno & PÚBLICO & Interno e Externo \\
\hline Espaço Real & LOCAL & Espaço real e virtual \\
\hline $\begin{array}{c}\text { Melhorias nas } \\
\text { habilidades }\end{array}$ & RESULTADOS & $\begin{array}{c}\text { Melhoria nos resultados } \\
\text { Passiva }\end{array}$ \\
$\begin{array}{c}\text { POSTURA } \\
\text { INDIVIDUAL }\end{array}$ & $\begin{array}{c}\text { Ativa (responsabilização pelo } \\
\text { autodesenvolvimento; aprender a } \\
\text { aprender) e contínua. }\end{array}$ \\
\hline $\begin{array}{c}\text { Pontuais ou } \\
\text { Fragmentadas }\end{array}$ & $\begin{array}{c}\text { AÇÕES NO } \\
\text { TEMPO }\end{array}$ & Contínuas \\
\hline
\end{tabular}

Fonte: Adaptado de Santos et al. (2001) confirmado por Aguilera (2020)

Percebe-se que a área de treinamento e desenvolvimento pode realizar uma capacitação específica dentro de uma área ou dentro de uma competência, desenvolvendo um novo curso de acordo com cada necessidade apontada. A diferença da universidade corporativa está na amplitude e na complexidade, geralmente na segunda opção é sempre mais abrangente do que, simplesmente, um treinamento. Pode-se dizer até que um programa de treinamento e desenvolvimento está atrelado as universidades corporativas, atendem aos objetivos estratégicos das organizações, mas nas UC's são abordados de forma mais completas.

\subsection{Universidades Corporativas: Oportunidades ou Ameaças às Universidades Tradicionais?}

Dentro do universo de mudanças, é possível identificar muitas oportunidades de desenvolvimento dentro das organizações, a aprendizagem contínua obriga, cada vez mais, a busca 
constante pelo desenvolvimento de competências, ajustes tecnológicos e facilidade no processo de aprendizagem. Dessa maneira, tudo que se refere às novas tecnologias e a educação continuada à distância tem alcançado destaque importante por se constituírem em uma modalidade de atualização, capacitação e formação profissional, que pode abranger diferentes níveis e áreas do conhecimento.

É preciso reconhecer que tais recursos tecnológicos tonaram-se instrumentos indispensáveis, no que se refere à autodidaxia, ocupando, desta maneira, vários projetos que se referem a educação da atual geração e, também, das futuras gerações. O que se reconhece atualmente, como educação continuada, tonou-se uma inevitável prática que surgiu a partir da rápida evolução científica e tecnológica (Veloso, 2004). Vidal e Miguel (2020, p. 03) relatam que:

O século XXI é sinônimo de mudanças significativas para a sociedade, com inúmeras transformações em todas as dimensões da vida humana, podendo ser citado dentro deste contexto evolutivo a expansão das mídias digitais onde esse progresso tecnológico é notável, reconfigurando a forma de organização social e profissional, maneiras de comunicação e a relação entre os indivíduos.

No caso das universidades tradicionais, estas trabalham dentro do processo singular no processo de aprendizagem. Realizam a formação do pensamento crítico do indivíduo e possibilitam o aperfeiçoamento da sua formação através do estágio prático nas organizações.

Eboli (1999) defende que o conceito de universidade corporativa promove uma significativa alteração na relação entre empresa e escola. Ressalta que com o aprimoramento constante deste conceito, mais e mais parcerias serão estabelecidas entre ambas. Sobre o conceito de educação corporativa Silva, Freitas e D'Ambrosio (2021, p. 03) falam o seguinte:

O conceito de Educação Corporativa (EC) passou a ser popularizado no final do século $X X$ em referência às práticas educacionais assumidas pelas organizações. Abrange uma imensa diversidade de modalidades, desde programas de treinamentos a educação formal, de todos os níveis, do ensino básico à formação específica, como cursos de graduação e pós-graduação, com métodos presenciais e a distância, e em parcerias com instituições de ensino superior (IES).

Ressalte-se que é possível identificar, como uma forte parceria utilizada pelas empresas nesse cenário de educação corporativa, já que os objetivos das universidades tradicionais são mais abrangentes conforme pode ser visualizado no quadro 2 . 
Quadro 2 - As Funções da Universidade Tradicional e da Universidade Corporativa

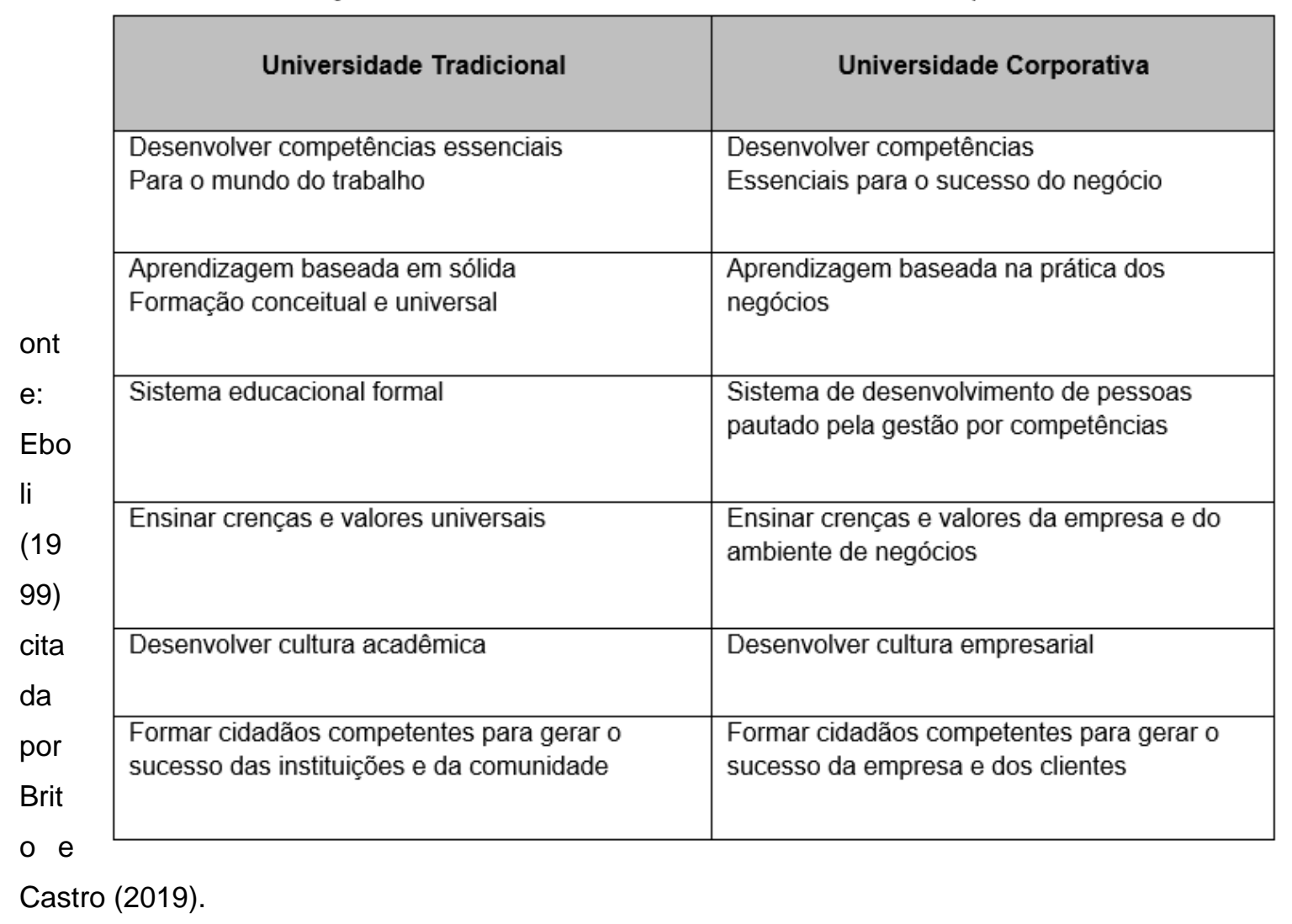

Percebe-se, naturalmente, que na medida que estão em um processo de atualização constante, as universidades corporativas $e$ as universidades tradicionais estão promovendo inovações, possibilitando, desta maneira, a aprendizagem e o desenvolvimento de todos, contribuindo e interagindo nesse processo. Portanto, não se pode determinar como sendo uma ameaça enquanto esse processo de atualização continuar acontecendo.

\section{CONSIDERAÇÕES FINAIS}

Entende-se que as universidades corporativas, através de ferramentas aliadas a tecnologias de educação á distancia, possibilitam um progresso importante sob vários aspectos. No cenário passado, ao se perceber a necessidade de instrução de funcionários para a melhoria da performance de habilidades operacionais específicas para as tarefas a serem realizadas, surgiu os centros de treinamentos, no entanto, com as mudanças no cenário das organizações e a competitividade juntamente com o crescimento tecnológico, dentro de uma gestão flexível, surgiu os primeiros sinais de educação corporativa.

A partir da década de 70 , surgiu a necessidade de ir além dos treinamentos convencionais para o desenvolvimento de habilidades, capacidades e atitudes dentro do cenário estratégico e tático das empresas, surgindo as universidades corporativas desenvolvendo as pessoas pautado pela gestão do conhecimento. Importante papel pautado na gestão de pessoas, que tem responsabilidade de promover e acompanhar todo o processo de aprendizagem dentro das organizações. 
Percebe-se sobre a mudança de paradigma no treinamento e desenvolvimento para as universidades corporativas, não descartando a importância da área de T\&D e sim, ressaltando a importância de focar na visão estratégica da empresa, investindo no aprendizado contínuo dos colaboradores através do desenvolvimento de competências dentro da visão organizacional. Nota-se a importância da utilização das tecnologias como ferramenta para dinamizar, ainda mais, suas formas de ensino e acesso a um número maior de pessoas, gerando mais aprendizado e desenvolvimento aos funcionários através de mídias alternativas. Evolução importante que representa o diferencial das universidades corporativas atualmente.

Por fim, o que é possível perceber sobre a questão das universidades corporativas representarem ameaças às universidades tradicionais, é que esta compreensão vem perdendo força pois, cada vez mais, identifica-se uma crescente associação de parceria através de programas educacionais em prol do desenvolvimento humano. Só seria de fato uma ameaça se as universidades tradicionais fossem, totalmente, fechadas ao estabelecimento de parcerias com as empresas em busca desse desenvolvimento. Sendo assim, dentro do formato atual que as universidades corporativas vêm crescendo, pode-se estreitar, ainda mais, o relacionamento com as universidades tradicionais nesse contínuo processo de atualização que acontece todos os dias.

\section{REFERÊNCIAS}

Aguilera, C. (2020). O que é Universidade Corporativa? Cuiabá: Grupo Gazeta. Disponível em: https://www.gazetadigital.com.br/colunas-e-opiniao/colunas-e-artigos/o-que-universidadecorporativa/604332. Acesso em: out/2021.

Araújo, U. V. (2018). Framework de uma universidade corporativa para alinhar competências com objetivos estratégicos e operacionais. Dissertação (Mestrado em Gestão Pública) UFES/ES. Espirito Santo: UFES/ES.

Brito, L. M. P., \& Castro, A. B. C. de. (2019). Universidades Corporativas: quando o saber construído pela humanidade é substituído pelo conhecimento empresarial. Innovar, 29(72), 147-162. ISSN 0121-5051. Doi: 10.15446/innovar.v29n72.77939.

Brizolla, M. M. B., Petry, J. F., Uchôa, A. G. F., \& Ferreira, H. L. B. (2020). Uma Revisão Sobre a Pesquisa Qualitativa em Ciências Sociais Aplicadas. UFAM Business Review, v. 2, n. 3, art. 6, pp. 103-130, julho-dezembro. ISSN: 2674-6581. DOI: https://10.47357/ufambr.v2i3.8087.

Eboli, M. (1999). Desenvolvimento e alinhamento dos talentos humanos às estratégias empresariais: o surgimento das universidades corporativas. São Paulo: Schmukles Editores.

Eboli, M. (2012). Educação corporativa no Brasil: evolução, conceitos e papéis. In: Casarini, F. G., Baumgartner, M. (Orgs.). Educação Corporativa: da teoria à prática. São Paulo: Editora Senac.

Esteves, L. P., \& Meirinõ, M. J. (2016). A Educação corporativa e a gestão do conhecimento. In: XI Congresso Nacional de Excelência em Gestão. Rio de Janeiro.

Falsarella, O. M., \& Jannuzzi, C. A. S. C. (2017). Planejamento estratégico empresarial e planejamento de tecnologia de informação e comunicação: uma abordagem utilizando 
projetos. Gestão e Produção, 24 (3), 610-621. ISSN: 0104-530X. Doi: 10.1590/0104530X481-16.

Fleury, M. T. L., \& Fleury, A. (2002). Construindo o conceito de competência. Revista de administração contemporânea, v. 5, n. SPE, p. 183-196. ISSN 1982-7849.

Gould, K. E. (2005). The corporate university: A model for sustaining an expert workforce in the human services. Behavior modification, v. 29, n. 3, p. 508-520. ISSN: 0145-4455.

Huhne, L. M. (1999). Metodologia científica. Rio de Janeiro: Vozes.

Leal, A. P., Bicalho, R. F. S., Sanchess, V. L., Melo, A. da S., Matos, S., Andrade, R. P., \& Magalhães, G. C. (2018). Gestão de competências: competências individuais x competências organizacionais. Revista Científica Multidisciplinar Núcleo do Conhecimento. Ano 03, Ed. 08, Vol. 10, pp. 72-88, agosto. ISSN:2448-0959.

Meister, J. C. (1999). Educação Corporativa. São Paulo: Makron Books.

Pessoa, D. S., Felix, G. S., \& Gomes, R. L. R. (2019). A importância da gestão por processos nos resultados corporativos. Revista Observatorio de la Economía Latinoamericana. Vol. 04. abril. 2019. ISSN: 1696-8352.

Silva, S. R. N. da. (2018). A Educação Corporativa Como Fonte de Vantagem Competitiva. Revista Semente, Ano 1, Número 1.

Silva, A. P. da., Freitas, F. C. H. P. de, \& D’Ambrósio, I. S. S. (2021). Educação Corporativa: análise crítico-discursiva da literatura especializada no campo acadêmico brasileiro. Discurso \& Sociedade, Vol.15(2). p.439-463. ISSN 1887-4606.

Silva, C. T. D. P. da, Nascimento, D. N. do, Silva, N. de S., \& Gomes, R. L. R. (2018). Educação corporativa como um fator de motivação. Revista Atlante: Cuadernos de Educación y Desarrollo. Vol. 03. março.. ISSN: 1989-4155.

Veloso, C. A. B. (2004). Análise das estratégias utilizadas em educação à distância nas organizações brasileiras. Estudo de caso em duas organizações de grande porte. Niterói: Universidade Federal Fluminense.

Vidal, A. S., \& Miguel, J. R. (2020). As Tecnologias Digitais na Educação Contemporânea. Rev. Mult. Psic. V.14, N. 50 p. 366-379, maio. ISSN 1981-1179. DOI: 10.14295/idonline.v14i50.2443. 